\title{
Orbits under a class of isometries of $L^{1}[0,1]$
}

\author{
by \\ TERJE HÕIM (Kent, OH)
}

\begin{abstract}
We study the orbits of isometries of $L^{1}[0,1]$. For a certain class of isometries we show that the set of functions $f$ in $L^{1}[0,1]$ for which the orbit of $f$ under the isometry $T$ is equivalent to the usual canonical basis $\left\{e_{1}, e_{2}, e_{3}, \ldots\right\}$ of $l^{1}$ is an open dense set. In the proof we develop a new method to get copies of $l^{1}$ inside $L^{1}[0,1]$ using geometric progressions. This method does not use disjoint or relatively disjoint supports, which seems to be the most common way to get such copies. We also use this method to prove a similar result for the shift operator on $l^{p}, 1 \leq p<\infty$. Finally, we study the orbits of multiplication operators on $H^{2}$ and $A(\mathbb{T})$, the set of all continuous complex-valued functions on $\mathbb{T}$ with absolutely convergent Fourier series.
\end{abstract}

Introduction. One of the fundamental problems in Operator Theory is the Invariant Subspace Problem, which asks whether every bounded linear operator on an infinite-dimensional Banach space admits a closed nontrivial invariant subspace. Recall that a subspace $Y$ of $X$ is invariant under $T: X \rightarrow X$ if $T Y \subset Y$. Most attempts so far have been made in the positive direction, that is, trying to prove that different classes of operators have nontrivial invariant subspaces. The study of invariant subspaces can be seen as a study of particular properties of orbits of operators in the following way: The orbit of an element $x \in X$ under the operator $T$ is the set of iterates $\left\{x, T x, T^{2} x, \ldots\right\}$. We say that a vector $x_{0} \in X$ is cyclic for $T$ provided the closed linear span of its orbit is the whole space, that is, $\overline{\operatorname{span}}\left\{x_{0}, T x_{0}, T^{2} x_{0}, \ldots\right\}=X$.

The question of whether an operator $T$ has nontrivial invariant subspaces is now equivalent to the question of whether all nonzero vectors $x$ are cyclic for $T$.

In recent years there has been a growing interest in studying the behavior of orbits in more detail. We mention here B. Beauzamy's book [1], the study

2000 Mathematics Subject Classification: Primary 47B38.

Key words and phrases: isometries, orbits of operators, shift operator, multiplication operator. 
of cyclic and hypercyclic operators, etc. In this paper we study the orbits of a class of isometries of $L^{1}[0,1]$.

Main theorem. By Lamperti [3], every isometry of $L^{1}$ can be written as $T f=h(f \circ \tau)$, where $\tau$ is a Borel measurable mapping of $[0,1]$ onto $[0,1]$ and $h \in L^{1}$. We will study the cases when the orbit of a function $f$ in $L^{1}$ under the isometry $T$ is equivalent to the usual canonical basis $\left\{e_{1}, e_{2}, e_{3}, \ldots\right\}$ of $l^{1}$. As we will see, there are essential differences depending on whether $\tau$ is measure preserving or not.

Proposition 1. If the point transformation $\tau$ determined by an isometry $T$ is measure preserving, then for every $f \in L^{1}[0,1]$ the closed subspace spanned by the orbit of $f$ under the isometry $T$ is not isomorphic to $l^{1}$.

Proof. We need the following definition: if $F$ is a subset of $L^{1}[0,1]$, then the functions in $F$ are called equi-integrable if

$$
\lim _{\alpha \rightarrow \infty} \sup _{f \in F} \int_{\{|f|>\alpha\}}|f(t)| d t=0 .
$$

Since $\left|T^{k} f(t)\right|=\left|f\left(\tau_{k}(t)\right)\right|$ for all $k \in \mathbb{N}$, where $|h(t)|=1$, the above limit is zero for all functions from the orbit of $f$. Hence orb $(T, f)=\left\{f, T f, T^{2} f, \ldots\right\}$ is equi-integrable. The topological characterization of equi-integrability is the following:

A subset $F$ of $L^{1}$ is equi-integrable if and only if it is relatively compact for $\sigma\left(L^{1}, L^{\infty}\right)$.

Hence, $\operatorname{orb}(T, f)=\left\{f, T f, T^{2} f, \ldots\right\}$ is relatively weakly compact. Kadec and Pełczyński [2] showed that $F \subset L^{1}$ is relatively weakly compact if and only if no sequence of elements of $F$ is equivalent to the unit vector basis of $l^{1}$. Hence $\operatorname{orb}(T, f)$ cannot span a subspace isomorphic to $l^{1}$.

We consider the following class of isometries $T$ on $L^{1}[0,1]$ :

$$
T f(x)= \begin{cases}\frac{1}{\lambda} f\left(\frac{x}{\lambda}\right) & \text { if } 0 \leq x \leq \lambda / 2, \\ \frac{1}{2-\lambda} f\left(\frac{1}{2-\lambda} x+\frac{1-\lambda}{2-\lambda}\right) & \text { if } \lambda / 2<x \leq 1,\end{cases}
$$

where $1 / 2 \leq \lambda<1$.

As one can see, the above isometry $T$ is determined by a point transformation mapping $\tau$ of $[0,1]$ into $[0,1]$ where $\tau$ is not measure preserving. More precisely, it "squeezes" some intervals and "stretches" others. The following theorem will show that in this case the orbits under $T$ are often $l^{1}$-bases.

THEOREM 2. Let $T$ be as in (1). The set of functions $f$ in $L^{1}[0,1]$ for which the orbit of $f$ under the isometry $T$ is equivalent to the usual canonical basis $\left\{e_{1}, e_{2}, e_{3}, \ldots\right\}$ of $l^{1}$ is an open dense set. 
Proof. Let $f \in L^{1}[0,1]$. Since the simple functions are dense in $L^{1}[0,1]$, for any $\varepsilon>0$ there is an $n \in \mathbb{N}$ and a simple function

$$
s=\sum_{k=1}^{2^{n}} s_{k} \chi_{\left[(k-1) / 2^{n}, k / 2^{n}\right]}
$$

such that $\|f-s\|<\varepsilon$. This form of a simple function assumes that the interval $[0,1]$ is divided into $2^{n}$ equal subintervals. Since the point transformation $\tau$, where

$$
\tau(x)= \begin{cases}\frac{x}{\lambda} & \text { if } 0 \leq x \leq \lambda / 2, \\ \frac{1}{2-\lambda} x+\frac{1-\lambda}{2-\lambda} & \text { if } \lambda / 2 \leq x \leq 1,\end{cases}
$$

determined by the isometry $T$ in (1), is not measure preserving, in order to simplify further formulas and computations, we will use the nature of $\tau$ and divide the interval $[0,1]$ into unequal subintervals in the following way: the first half $[0,1 / 2]$ is divided by a refinement of the dissection $\lambda^{n-1} / 2, \lambda^{n-2} / 2$, $\lambda^{n-3} / 2, \ldots, \lambda / 2,1 / 2$ into $(n-1) 2^{n-1}+1$ subintervals

$$
\begin{aligned}
& {\left[0, \frac{\lambda^{n-1}}{2}\right]} \\
& \quad \cup \bigcup_{k=1}^{n-1} \bigcup_{j=1}^{2^{n-1}}\left[\frac{\lambda^{n-k}}{2}+(j-1) \frac{\lambda^{n-k-1}-\lambda^{n-k}}{2^{n}}, \frac{\lambda^{n-k}}{2}+j \frac{\lambda^{n-k-1}-\lambda^{n-k}}{2^{n}}\right],
\end{aligned}
$$

the second half $[1 / 2,1]$ is divided by a refinement of the dissection $1 / 2,1-$ $1 /(2(2-\lambda)), 1-1 /\left(2(2-\lambda)^{2}\right), \ldots, 1-1 /\left(2(2-\lambda)^{n-1}\right), 1$ into $(2 n-4) 2^{n-1}+1$ subintervals

$$
\begin{aligned}
& \bigcup_{k=1}^{2 n-6} \bigcup_{j=1}^{2^{n-1}}\left[1-\frac{1}{2(2-\lambda)^{k}}+(j-1) \frac{1-\lambda}{2^{n}(2-\lambda)^{k+1}},\right. \\
& \left.1-\frac{1}{2(2-\lambda)^{k}}+j \frac{1-\lambda}{2^{n}(2-\lambda)^{k+1}}\right] \\
& \cup\left[1-\frac{1}{2(2-\lambda)^{2 n-5}}, 1-\frac{1}{2(2-\lambda)^{2 n-4}}\right] \cup\left[1-\frac{1}{2(2-\lambda)^{2 n-4}}, 1\right] .
\end{aligned}
$$

In this way we will have more subintervals than needed, but it will be easier to describe and understand how the point transformation $\tau$ works. Namely, $\tau$ will take each "big" interval $\left[\lambda^{n-k} / 2, \lambda^{n-k-1} / 2\right], k=1, \ldots, n-1$, of $[0,1 / 2]$, and $\left[1-1 /\left(2(2-\lambda)^{k}\right), 1-1 /\left(2(2-\lambda)^{k+1}\right)\right], k=1, \ldots, 2 n-5$, of $[1 / 2,1]$, directly to the left of it in a 1-1 fashion and the endpoints of one interval will be mapped to the endpoints of another interval. Each of the small subintervals will therefore be mapped $2^{n-1}$ intervals to the left. The only intervals that do not get moved around are the very first and 
the very last interval of $[0,1]$. The interval $\left[0, \lambda^{n-1} / 2\right]$ will be squeezed and mapped onto the $\lambda$ multiple of itself, that is, onto $\left[0, \lambda^{n} / 2\right]$, and the very last interval $\left[1-1 / 2(2-\lambda)^{2 n-4}, 1\right]$ will be stretched and mapped onto $\left[1-1 /\left(2(2-\lambda)^{2 n-5}\right), 1\right]$. The function $s$ is constant on each of these intervals.

We will prove the equivalence to the canonical basis for the orbit of an arbitrary simple function assuming that the interval $[0,1]$ is subdivided as in (3). Let a simple function $s$ have a constant value $s_{k}$ on the $k$ th subinterval of the division (3), $1 \leq k \leq(3 n-6) 2^{n-1}+2$. We apply the isometry $T$ to this simple function $m$ times, where $m \in \mathbb{N}$. We will show that there are constants $c_{1}, c_{2}>0$ such that

$$
c_{1} \sum_{k=0}^{m}\left|a_{k}\right| \leq\left\|\sum_{k=0}^{m} a_{k} T^{k} s\right\|_{L^{1}} \leq c_{2} \sum_{k=0}^{m}\left|a_{k}\right|
$$

for any sequence $\left(a_{k}\right)$ of complex numbers in $l^{1}$ and for any $m \in \mathbb{N}$. This means that there is an isomorphism of $l^{1}$ onto the closed subspace of $L^{1}[0,1]$ spanned by the orbit of $s$ under the isometry $T$. Under this isomorphism the unit vectors in $l^{1}$ correspond to the functions $T^{k} s$. In other words, the sequence $\left\{s, T s, T^{2} s, \ldots\right\}$ is equivalent to the unit vector basis in $l^{1}$.

The right hand side inequality with $c_{2}=1$ (or $c_{2}=\|s\|_{L^{1}}$ if $\|s\|_{L^{1}} \neq 1$ ) in (4) follows easily from the fact that if $s$ has $L^{1}$-norm 1 , then since $T$ is an isometry, $\left\|T^{k} s\right\|=1$ for any $k \in \mathbb{N}$. Therefore, for any $a_{0}, \ldots, a_{m}$, we have

$$
\begin{aligned}
\left\|\sum_{k=0}^{m} a_{k} T^{k} s\right\|_{L^{1}} & =\int_{0}^{1}\left|\sum_{k=0}^{m} a_{k} T^{k} s(t)\right| d t \leq \int_{0}^{1} \sum_{k=0}^{m}\left|a_{k}\right|\left|T^{k} s(t)\right| d t \\
& =\sum_{k=0}^{m}\left|a_{k}\right| \int_{0}^{1}\left|T^{k} s(t)\right| d t=\sum_{k=0}^{m}\left|a_{k}\right| .
\end{aligned}
$$

In order to get the left hand inequality in (4), we need to write up the $L^{1}$-norm of $\sum_{k=0}^{m} a_{k} T^{k} s$ very precisely. Since $s$ is a simple function, $\int\left|\sum a_{k} T^{k} s(t)\right| d t$ is the sum of the areas of the rectangles with the above subintervals (3) as bases and the corresponding linear combinations $\left|\sum a_{k} T^{k} s(t)\right|$ of the $s_{i}$ 's as heights. For example, the first two rectangles have the same base $\lambda^{n+m-1} / 2$ but

$$
\left|\sum_{k=0}^{m} \frac{1}{\lambda^{k}} a_{k} s_{1}\right| \text { and }\left|\sum_{k=0}^{m-1} \frac{1}{\lambda^{k}} a_{k} s_{1}+\frac{1}{\lambda^{m}} a_{m} s_{2}\right|
$$

as their respective heights. Two consecutive rectangles on either side of the dissection point $\lambda^{n-1} / 2$ have widths $\lambda^{n-1} / 2^{n}$ and $\left(\lambda^{n-2}-\lambda^{n-1}\right) / 2^{n}$, and heights 


$$
\begin{aligned}
\mid \sum_{k=0}^{n-1} \frac{1}{\lambda^{k}} a_{k} s_{k 2^{n-1}}+\sum_{k=n}^{3 n-6} \frac{1}{\lambda^{n-1}} \frac{1}{(2-\lambda)^{k-n+1}} a_{k} s_{k 2^{n-1}} \\
+\frac{1}{\lambda^{n-1} \frac{1}{(2-\lambda)^{2 n-4}} a_{3 n-5} s_{(3 n-6) 2^{n-1}+1}} \\
+\sum_{k=3 n-4}^{m} \frac{1}{\lambda^{n-1}} \frac{1}{(2-\lambda)^{k-n+1}} a_{k} s_{(3 n-6) 2^{n-1}+2} \mid
\end{aligned}
$$

and

$$
\begin{aligned}
\mid \sum_{k=0}^{n-2} \frac{1}{\lambda^{k}} a_{k} s_{k 2^{n-1}+1} & +\sum_{k=n-1}^{3 n-6} \frac{1}{\lambda^{n-2}} \frac{1}{(2-\lambda)^{k-n+2}} a_{k} s_{k 2^{n-1}+1} \\
& +\sum_{k=3 n-5}^{m} \frac{1}{\lambda^{n-2}} \frac{1}{(2-\lambda)^{k-n+2}} a_{k} s_{(3 n-6) 2^{n-1}+2} \mid
\end{aligned}
$$

respectively. And so on. The $L^{1}$-norm of $\sum_{k=0}^{m} a_{k} T^{k} s$ is a sum of $(3 n-6) 2^{n-1}$ $+m+3$ terms similar to these four above. This sum is, of course, very lengthy, but if we use the triangle inequality $|a|+|b| \geq|b-a|$ to compare each pair of consecutive terms in this sum and pick only those terms that we get if we compare the areas of two consecutive rectangles whose base intervals have a common endpoint at $\lambda^{k} / 2, k=1, \ldots, n+m-1$, in $[0,1 / 2]$ or at $1-1 /\left(2(2-\lambda)^{k}\right), k=1, \ldots, 2 n-4$, in $(1 / 2,1]$, then the sum we end up with will have a very simple structure. In particular, we get

$$
\left\|\sum_{k=0}^{m} a_{k} T^{k} s\right\| \geq \frac{1}{2^{n}}\left(\frac{1-\lambda}{2^{n}}\right)\left(S_{1}+S_{2}+S_{3}\right),
$$

where $S_{1}=S_{1}\left(a_{0}, \ldots, a_{3 n-6}\right), S_{2}=S_{2}\left(a_{0}, \ldots, a_{m}\right)$ and $S_{3}=S_{3}\left(a_{m-3 n+6}\right.$, $\left.\ldots, a_{m}\right)$ are defined as follows:

$$
\begin{aligned}
S_{1}= & \left|a_{0} w_{3 n-4}\right|+\left|a_{0} w_{3 n-5}+a_{1} w_{3 n-4}\right| \\
& +\left|a_{0} w_{3 n-6}+a_{1} w_{3 n-5}+a_{2} w_{3 n-4}\right|+\ldots \\
& +\left|a_{0} w_{2}+a_{1} w_{3}+\ldots+a_{3 n-6} w_{3 n-4}\right| ; \\
S_{2}= & \left|a_{0} w_{1}+a_{1} w_{2}+\ldots+a_{3 n-5} w_{3 n-4}\right|+ \\
& +\left|a_{1} w_{1}+a_{2} w_{2}+\ldots+a_{3 n-4} w_{3 n-4}\right| \\
& +\left|a_{2} w_{1}+a_{3} w_{2}+\ldots+a_{3 n-3} w_{3 n-4}\right|+\ldots \\
& +\left|a_{m-3 n+5} w_{1}+a_{m-3 n+6} w_{2}+\ldots+a_{m} w_{3 n-4}\right| ; \\
S_{3}= & \left|a_{m-3 n+6} w_{1}+a_{m-3 n+7} w_{2}+\ldots+a_{m} w_{3 n-5}\right| \\
& +\left|a_{m-3 n+7} w_{1}+a_{m-3 n+8} w_{2}+\ldots+a_{m} w_{3 n-6}\right| \\
& +\ldots+\left|a_{m-1} w_{1}+a_{m} w_{2}\right|+\left|a_{m} w_{1}\right|,
\end{aligned}
$$


with a vector $w \in \mathbb{R}^{3 n-4}$, where

$$
\begin{aligned}
w_{1} & =\lambda^{n-1}\left(s_{1}-s_{0}\right) \\
w_{2} & =\lambda^{n-2}\left(s_{2^{n-1}+1}-s_{2^{n-1}}\right) \\
w_{3} & =\lambda^{n-3}\left(s_{2 \cdot 2^{n-1}+1}-s_{2 \cdot 2^{n-1}}\right), \ldots, \\
w_{n-1} & =\lambda\left(s_{(n-2) 2^{n-1}+1}-s_{\left.(n-2) 2^{n-1}\right)}\right. \\
w_{n} & =\frac{\lambda}{2-\lambda} s_{(n-1) 2^{n-1}+1}-s_{(n-1) 2^{n-1}} \\
w_{n+1} & =\frac{1}{2-\lambda}\left(\frac{\lambda}{2-\lambda} s_{n 2^{n-1}+1}-s_{n 2^{n-1}}\right), \ldots, \\
w_{3 n-5} & =\frac{1}{(2-\lambda)^{2 n-5}}\left(\frac{\lambda}{2-\lambda} s_{(3 n-6) 2^{n-1}+1}-s_{(3 n-6) 2^{n-1}}\right) \\
w_{3 n-4} & =\frac{1}{(2-\lambda)^{2 n-4}}\left(\frac{\lambda}{2-\lambda} s_{(3 n-6) 2^{n-1}+2}-s_{(3 n-6) 2^{n-1}+1}\right) .
\end{aligned}
$$

Since we are proving a statement for a dense set of functions, we can always perturb the simple function $s$ a little so that not all of the $w_{i}$ 's are zero.

Thus, our final task is to estimate the sum $S_{1}+S_{2}+S_{3}$. In order to do that, we need the following proposition which we feel is interesting in itself, and which shows that $S_{2}$ is small under some conditions.

Proposition 3. Fix $n \in \mathbb{N}$. There is a dense set of vectors $w=$ $\left(w_{1}, \ldots, w_{n+1}\right)$ in $\mathbb{C}^{n+1}, w_{1} \neq 0, w_{n+1} \neq 0$, with the following property: if $a$ sequence $\left(\alpha_{k}\right)$ of complex numbers satisfies $\left\langle w, A_{i}\right\rangle=0$ for all $i=0,1,2, \ldots$, where $A_{i}=\left(\alpha_{i}, \alpha_{i+1}, \ldots, \alpha_{i+n}\right)$, then $\left(\alpha_{k}\right)$ can be written as a linear combination of $n$ geometric progressions.

Proof. Take a vector $w^{\prime}=\left(w_{1}^{\prime}, \ldots, w_{n+1}^{\prime}\right)$ with $w_{1}^{\prime} \neq 0$ and $w_{n+1}^{\prime} \neq 0$. If the $n$ th-degree polynomial $p(y)=w_{n+1}^{\prime} y^{n}+w_{n}^{\prime} y^{n-1}+\ldots+w_{2}^{\prime} y+w_{1}^{\prime}$ does not have distinct roots, find another vector $w=\left(w_{1}, \ldots, w_{n+1}\right)$, very close to $w^{\prime}$, such that the above $n$ th-degree polynomial with new coefficients $w_{1}, \ldots, w_{n+1}$ has $n$ distinct roots $y_{1}, \ldots, y_{n}$.

Let $\left(\alpha_{k}\right)$ be a sequence of complex numbers such that $\left\langle w, A_{i}\right\rangle=0$ for all $i=0,1,2, \ldots$ To prove the proposition, suppose that the first $n$ numbers $\alpha_{0}, \alpha_{1}, \ldots, \alpha_{n-1}$ can be written in the following form:

$$
\begin{aligned}
x_{1}+x_{2}+\ldots+x_{n} & =\alpha_{0}, \\
x_{1} y_{1}+x_{2} y_{2}+\ldots+x_{n} y_{n} & =\alpha_{1}, \\
x_{1} y_{1}^{2}+x_{2} y_{2}^{2}+\ldots+x_{n} y_{n}^{2} & =\alpha_{2}, \\
\vdots & \\
x_{1} y_{1}^{n-1}+x_{2} y_{2}^{n-1}+\ldots+x_{n} y_{n}^{n-1} & =\alpha_{n-1} .
\end{aligned}
$$


Since $y_{1}, \ldots, y_{n}$ are distinct, the Vandermonde determinant of $\left\{1, y_{1}, \ldots, y_{n}\right\}$ is nonzero and therefore there are unique $x_{1}, \ldots, x_{n}$ solving the system (7).

By assumption, $\alpha_{0} w_{1}+\alpha_{1} w_{2}+\ldots+\alpha_{n} w_{n+1}=0$, so

$$
\begin{aligned}
\alpha_{n}= & -\alpha_{0} \frac{w_{1}}{w_{n+1}}-\alpha_{1} \frac{w_{2}}{w_{n+1}}-\ldots-\alpha_{n-1} \frac{w_{n}}{w_{n+1}} \\
= & \left(-\frac{w_{1}}{w_{n+1}}\right)\left(x_{1}+\ldots+x_{n}\right)+\left(-\frac{w_{2}}{w_{n+1}}\right)\left(x_{1} y_{1}+\ldots+x_{n} y_{n}\right) \\
& +\ldots+\left(-\frac{w_{n}}{w_{n+1}}\right)\left(x_{1} y_{1}^{n-1}+\ldots+x_{n} y_{n}^{n-1}\right) \\
= & x_{1}\left(-\frac{w_{1}}{w_{n+1}}-\frac{w_{2}}{w_{n+1}} y_{1}-\frac{w_{3}}{w_{n+1}} y_{1}^{2}-\ldots-\frac{w_{n}}{w_{n+1}} y_{1}^{n-1}\right) \\
& +x_{2}\left(-\frac{w_{1}}{w_{n+1}}-\frac{w_{2}}{w_{n+1}} y_{2}-\ldots-\frac{w_{n}}{w_{n+1}} y_{2}^{n-1}\right) \\
& +\ldots+x_{n}\left(-\frac{w_{1}}{w_{n+1}}-\frac{w_{2}}{w_{n+1}} y_{n}-\ldots-\frac{w_{n}}{w_{n+1}} y_{n}^{n-1}\right) \\
= & x_{1} y_{1}^{n}+x_{2} y_{2}^{n}+\ldots+x_{n} y_{n}^{n},
\end{aligned}
$$

because $y_{1}, \ldots, y_{n}$ were exactly the $n$ roots of the $n$ th-degree polynomial $p(y)=w_{n+1} y^{n}+w_{n} y^{n-1}+\ldots+w_{2} y+w_{1}$. Note that $y_{1}, \ldots, y_{n}$ are uniquely determined by $w_{1}, \ldots, w_{n+1}$.

Next, if $\alpha_{1} w_{1}+\alpha_{2} w_{2}+\ldots+\alpha_{n+1} w_{n+1}=0$, then

$$
\begin{aligned}
\alpha_{n+1}= & -\frac{w_{1}}{w_{n+1}} \alpha_{1}-\frac{w_{2}}{w_{n+1}} \alpha_{2}-\ldots-\frac{w_{n}}{w_{n+1}} \alpha_{n} \\
= & -\frac{w_{1}}{w_{n+1}}\left(x_{1} y_{1}+\ldots+x_{n} y_{n}\right)-\frac{w_{2}}{w_{n+1}}\left(x_{1} y_{1}^{2}+\ldots+x_{n} y_{n}^{2}\right) \\
& -\ldots-\frac{w_{n}}{w_{n+1}}\left(x_{1} y_{1}^{n}+\ldots+x_{n} y_{n}^{n}\right) \\
= & x_{1} y_{1}^{n+1}+\ldots+x_{n} y_{n}^{n+1} .
\end{aligned}
$$

By (8) and (9) and by obvious induction we see that $\left(\alpha_{k}\right)$ can be written as a linear combination of $n$ geometric progressions.

Denote the geometric progressions from Proposition 3 by $g^{1}, \ldots, g^{n}$, where $g^{k}=\left(1, y_{k}, y_{k}^{2}, \ldots\right)$ for $k=1, \ldots, n$. Thus, every simple function $s$ gives us a vector $w \in \mathbb{R}^{3 n-4}$ as in (6) and a finite number of geometric progressions $g^{1}, g^{2}, \ldots, g^{3 n-5}$. Depending on these geometric series, we consider three possible cases:

CASE I: $g^{1}, \ldots, g^{3 n-5}$ are all decreasing.

CASE II: $g^{1}, \ldots, g^{3 n-5}$ are all increasing.

CASE III: some $g^{1}, \ldots, g^{3 n-5}$ are decreasing and some are increasing. 
Before considering these cases, assume that we have already shown that there is a dense set of functions $f$ for which there is a $\gamma>0$ such that $\left\|\sum_{k=0}^{m} a_{k} T^{k} f\right\|_{L^{1}} \geq \gamma \sum_{k=0}^{m}\left|a_{k}\right|$ for every $m \in \mathbb{N}$ and for every sequence of scalars $\left(a_{k}\right) \in l^{1}$. We will now show that this set is also open. Indeed, if $\|g-f\|<\gamma / 2$, then

$$
\begin{aligned}
\left\|\sum_{k=0}^{m} a_{k} T^{k} g\right\| & \geq\left\|\sum_{k=0}^{m} a_{k} T^{k} f\right\|-\left\|\sum_{k=0}^{m} a_{k} T^{k}(g-f)\right\| \\
& \geq \gamma \sum_{k=0}^{m}\left|a_{k}\right|-\frac{\gamma}{2} \sum_{k=0}^{m}\left|a_{k}\right|=\frac{\gamma}{2} \sum_{k=0}^{m}\left|a_{k}\right|,
\end{aligned}
$$

that is, also $\left\{g, T g, T^{2} g, \ldots\right\}$ is equivalent to the unit vector basis of $l^{1}$.

Thus, Theorem 2 will be proved once we show the existence of the above $\gamma>0$ in all three different cases.

CASE I. First we will consider the case when $\left|y_{k}\right|<1$ for all $k=$ $1,2, \ldots, 3 n-5$. Since the geometric progressions $g^{1}, \ldots, g^{3 n-5}$ are all decreasing, the following lemma will find a "universal block length" $M$ for all those $\alpha_{i}$ 's that are exact linear combinations of the geometric progressions $g^{1}, \ldots, g^{3 n-5}$ in such a way that the size of any $M$-block of $\alpha_{i}$ 's is less than $\frac{1}{10}$ th of the preceding $M$-block.

Lemma 4. There exists an $M>0$ depending only on $g^{1}, \ldots, g^{3 n-5}$ such that if $\left(\alpha_{i}\right) \in l^{1}$ is any sequence that can be written as $\left(\alpha_{i}\right)_{i \in \mathbb{N}}=\left(x_{1} g^{1}+\right.$ $\left.\ldots+x_{3 n-5} g^{3 n-5}\right)$, where $g^{k}=\left(1, y_{k}, y_{k}^{2}, \ldots\right), 1 \leq k \leq 3 n-5$, with $\left|y_{k}\right|<1$ and $y_{k} \neq y_{j}, 1 \leq k, j \leq 3 n-5, k \neq j$, then

$$
\sum_{i=M}^{2 M-1}\left|\alpha_{i}\right| \leq \frac{1}{10} \sum_{i=0}^{M-1}\left|\alpha_{i}\right|
$$

Proof. Since all the $y_{k}$ 's, $1 \leq k \leq 3 n-5$, are distinct, the Vandermonde determinant of $\left\{1, y_{1}, \ldots, y_{3 n-5}\right\}$ is nonzero. Hence $g^{1}, \ldots, g^{3 n-5}$ are linearly independent and form a basis for a $(3 n-5)$-dimensional subspace of $l^{1}$ with basis constant $K$. Consequently, for any $M$,

$$
\sum_{k=0}^{M-1}\left|\alpha_{k}\right|=\left\|x_{1} g^{1}+\ldots+x_{3 n-5} g^{3 n-5}\right\| \geq \frac{1}{K} \max _{1 \leq k \leq 3 n-5}\left|x_{k}\right| .
$$

On the other hand,

$$
\begin{aligned}
\sum_{k=M}^{2 M-1}\left|\alpha_{k}\right| & =\left\|x_{1} y_{1}^{M} g^{1}+\ldots+x_{3 n-5} y_{3 n-5}^{M} g^{3 n-5}\right\| \\
& \leq(3 n-5) \max _{1 \leq k \leq 3 n-5}\left|x_{k}\right| \max _{1 \leq k \leq 3 n-5}\left|y_{k}^{M}\right| .
\end{aligned}
$$


If we choose $M \in \mathbb{N}$ so large that

$$
\frac{1}{K(3 n-5) \max _{1 \leq k \leq 3 n-5}\left|y_{k}^{M}\right|} \geq 10,
$$

then

$$
\begin{aligned}
\sum_{i=0}^{M-1}\left|\alpha_{i}\right| & =\left\|x_{1} g^{1}+\ldots+x_{3 n-5} g^{3 n-5}\right\| \geq \frac{1}{K} \max _{1 \leq k \leq 3 n-5}\left|x_{k}\right| \\
& \geq \frac{1}{K(3 n-5) \max _{1 \leq k \leq 3 n-5}\left|y_{k}^{M}\right|}\left\|x_{1} y_{1}^{M} g^{1}+\ldots+x_{3 n-5} y_{3 n-5}^{M} g^{3 n-5}\right\| \\
& \geq 10\left\|x_{1} y_{1}^{M} g^{1}+\ldots+x_{3 n-5} y_{3 n-5}^{M} g^{3 n-5}\right\|=\sum_{i=M}^{2 M-1}\left|\alpha_{i}\right|
\end{aligned}
$$

Hence the desired $M$ exists and this proves the lemma.

Let the $\alpha_{i}$ 's, $y_{i}$ 's and $M$ be as above. Divide the integers into blocks of length $M$ and consider now any sequence $\left(a_{k}\right)$ of complex numbers. Corresponding to each $M$-block of integers consider the block of consecutive $a_{i}$ 's with indices from the $M$-block of integers. Let $\mathcal{A}_{j}$ be the block $\left(a_{(j-1) M}, \ldots, a_{j M-1}\right)$. We make the following

Definition 5 . When

$$
\sum_{i=j M}^{(j+1) M-1}\left|a_{i}\right| \leq \frac{1}{2} \sum_{i=(j-1) M}^{j M-1}\left|a_{i}\right|
$$

we say that the $a_{i}$ 's go down in the block $\mathcal{A}_{j+1}$. Otherwise they go up in $\mathcal{A}_{j+1}$.

We are looking for a $\gamma>0$ such that $S_{1}+S_{2}+S_{3} \geq \gamma \sum_{k=0}^{m}\left|a_{k}\right|$ for every $m \in \mathbb{N}$ and $\left(a_{k}\right) \in l^{1}$. In the case $S_{2}\left(a_{0}, \ldots, a_{m}\right)=0$, we get the lower estimate in the following way:

LemMA 6. Let $0 \neq \sum_{k=0}^{\infty}\left|a_{k}\right|<\infty$. If $S_{2}\left(a_{0}, \ldots, a_{m}\right)=0$ and $\left(a_{0}, \ldots, a_{m}\right)$ can be written as a linear combination of $3 n-5$ decreasing geometric progressions, then there exist $\delta_{1}>0$ and $\gamma_{1}>0$, depending only on those progressions, such that

$$
S_{1}\left(a_{0}, \ldots, a_{3 n-6}\right) \geq \delta_{1},
$$

and, consequently,

$$
\left(S_{1}+S_{2}+S_{3}\right)\left(a_{0}, \ldots, a_{m}\right) \geq \gamma_{1} \sum_{k=0}^{m}\left|a_{k}\right| .
$$

Proof. Suppose to the contrary that $S_{1}\left(a_{0}, \ldots, a_{3 n-6}\right)=0$. This implies that $\left|a_{0} w_{3 n-4}\right|=0$, and since $w_{3 n-4} \neq 0$, we must have $a_{0}=0$. Next, $\left|a_{0} w_{3 n-5}+a_{1} w_{3 n-4}\right|=\left|a_{1} w_{3 n-4}\right|=0$ implies that $a_{1}=0$. Continuing, we 
see that all of $a_{0}, \ldots, a_{m}$ must be zeros, contradicting the hypothesis that $\sum_{k=0}^{m}\left|a_{k}\right| \neq 0$. Hence $S_{1}\left(a_{0}, \ldots, a_{3 n-6}\right)$ has to be strictly positive. Since $S_{1}\left(a_{0}, \ldots, a_{3 n-6}\right)$ is a continuous function, and the set

$$
\left\{\left(a_{0}, \ldots, a_{M-1}\right): S_{2}\left(a_{0}, \ldots, a_{M-1}\right)=0 \text { and } \sum_{k=0}^{M-1}\left|a_{k}\right|=1\right\}
$$

is a compact subset of the unit sphere of $\mathbb{C}^{M}$, the function $S_{1}\left(a_{0}, \ldots, a_{3 n-6}\right)$ must attain its strictly positive minimum on this compact set. That is, there exists a $\delta_{1}>0$ such that $S_{1}\left(a_{0}, \ldots, a_{3 n-6}\right) \geq \delta_{1}$. Since $\sum_{k=0}^{m}\left|a_{k}\right| \leq$ $\frac{10}{9} \sum_{k=0}^{M-1}\left|a_{k}\right|$, for $\gamma_{1}=9 \delta_{1} / 10$ we get

$$
\left(S_{1}+S_{2}+S_{3}\right)\left(a_{0}, \ldots, a_{m}\right) \geq \delta_{1} \sum_{k=0}^{M-1}\left|a_{k}\right| \geq \gamma_{1} \sum_{k=0}^{m}\left|a_{k}\right|
$$

for all $m \in \mathbb{N}$ and for all sequences $\left(a_{k}\right)$ with $S_{2}\left(\left(a_{k}\right)\right)=0$.

If $\sum_{i=0}^{M-1}\left|a_{i}\right| \neq 1$, one can use the homogeneity of the absolute value to finish the proof of Lemma 6.

So, if Case I occurs and $S_{2}=0$, the equivalence from Theorem 2 is proved.

REMARK. Notice that if $S_{2} \neq 0$, then $S_{1}+S_{2} \neq 0$, and using the continuity of $S_{1}+S_{2}$ on the compact subset of the unit sphere of $\mathbb{C}^{M}$, a lower estimate follows.

In the following we assume that $S_{2} \neq 0$. Put

$$
\left\|\mathcal{A}_{j}\right\|=\sum_{i=(j-1) M}^{j M-1}\left|a_{i}\right| .
$$

Lemma 7 will give us a partial estimate involving any three consecutive blocks. All blocks are put together in Subcases I.1 and I.2. Lemma 8 will state the final result in Case I.

So, we still assume that the vector $w=\left(w_{1}, \ldots, w_{3 n-4}\right)$ gives us $3 n-5$ decreasing geometric progressions.

Lemma 7. There exists a $\gamma_{2}>0$, depending only on a given vector $w$, such that if $\left\|\mathcal{A}_{j}\right\| \leq \frac{1}{2}\left\|\mathcal{A}_{j-1}\right\|$, but $\left\|\mathcal{A}_{j+1}\right\|>\frac{1}{2}\left\|\mathcal{A}_{j}\right\|$, then

$$
\left(S_{1}+S_{2}+S_{3}\right)\left(a_{(j-1) M}, \ldots, a_{(j+1) M-1}\right) \geq \gamma_{2} \sum_{i=(j-1) M}^{(j+1) M-1}\left|a_{i}\right| .
$$

Proof. Since the $a_{i}$ 's go up in the block $\mathcal{A}_{j+1}$, we have $\left(a_{(j-1) M}, \ldots, a_{(j+1) M-1}\right)=x_{1} y_{1}^{(j-1) M} g^{1}+\ldots+x_{3 n-5} y_{3 n-5}^{(j-1) M} g^{3 n-5}+\eta^{2 M}$, 
where $g^{k}=\left(1, y_{k}, y_{k}^{2}, \ldots, y_{k}^{2 M-1}\right), 1 \leq k \leq 3 n-5$, and $\eta^{2 M}=\left(\eta_{(j-1) M}, \ldots\right.$ $\left.\ldots, \eta_{(j+1) M-1}\right)$ with $\left\|\eta^{2 M}\right\| \geq \varepsilon_{0}$, where $\varepsilon_{0}>0$ is determined by $g^{1}, \ldots, g^{3 n-5}$ and by combining Lemma 4 and Definition 5. Clearly, $S_{2}\left(a_{j M}, \ldots, a_{(j+1) M-1}\right) \neq 0$ for any such collection of $a_{j M}, \ldots, a_{(j+1) M-1}$. Since $S_{2}\left(a_{j M}, \ldots, a_{(j+1) M-1}\right)$ is a continuous function on a compact subset $\left\{\left(a_{j M}, \ldots, a_{(j+1) M-1}\right):\left\|\mathcal{A}_{j+1}\right\| \geq \frac{1}{2}\left\|\mathcal{A}_{j}\right\|\right.$ and $\left.\sum_{k=j M}^{(j+1) M-1}\left|a_{k}\right|=1\right\}$ of the unit sphere of $\mathbb{C}^{M}$, there is an $\varepsilon_{1}>0$ such that $S_{2}\left(a_{j M}, \ldots, a_{(j+1) M-1}\right) \geq \varepsilon_{1}$. Since

$$
\sum_{k=(j-1) M}^{(j+1) M-1}\left|a_{k}\right| \leq 3 \sum_{k=j M}^{(j+1) M-1}\left|a_{k}\right|,
$$

taking $\gamma_{2}=\varepsilon_{1} / 3$, we get the desired estimate.

We will now use Lemmas 6 and 7 to prove Lemma 8 and thus the equivalence from Theorem 2 in the case of decreasing geometric progressions.

Consider any sequence $\left(a_{k}\right)$ of complex numbers in $l^{1}$ and divide $\left(a_{k}\right)$ into blocks of length $M$ as before. We now give a label to each block-either "d" if the $a_{i}$ 's go down in that block or " $\mathrm{u}$ " if the $a_{i}$ 's go up there.

Depending on the sequence $\left(a_{k}\right)$, we need to consider the following two subcases:

SubCASE I.1: There is no block with label " $u$ ".

Then either Lemma 6 or the Remark following it gives us an estimate.

SuBCASE I.2: There is a block with label " $\mathrm{u}$ ".

Then for each block with " $u$ " consider the previous block and all consecutive blocks with "d" up to but not including the next "u"-block. Consider the blocks $\mathcal{A}_{j-1}, \mathcal{A}_{j}, \ldots, \mathcal{A}_{j+k}$, where $\mathcal{A}_{j-1}$ has either "u" or "d", $\mathcal{A}_{j}$ has "u" and $\mathcal{A}_{j+1}, \ldots \mathcal{A}_{j+k}$ all have "d". Then, by Lemmas 7 and 6 we have the following estimates:

$$
\begin{aligned}
& \left(S_{1}+S_{2}+S_{3}\right)\left(a_{(j-2) M}, \ldots, a_{j M-1}\right) \geq \gamma_{2} \sum_{i=(j-2) M}^{j M-1}\left|a_{i}\right| \\
& \left(S_{1}+S_{2}+S_{3}\right)\left(a_{j M}, \ldots, a_{(j+k) M-1}\right) \geq \gamma_{3} \sum_{i=j M}^{(j+k) M-1}\left|a_{i}\right| .
\end{aligned}
$$

Since

$$
\sum_{i=(j-1) M}^{(j+k) M-1}\left|a_{i}\right| \leq 2 \sum_{i=(j-1) M}^{j M-1}\left|a_{i}\right|
$$

we get 


$$
\begin{array}{r}
\left(S_{1}+S_{2}+S_{3}\right)\left(a_{(j-2) M}, \ldots, a_{(j+k) M-1}\right) \geq \gamma_{2} \sum_{i=(j-2) M}^{(j-1) M-1}\left|a_{i}\right|+\gamma_{2} \sum_{i=(j-1) M}^{j M-1}\left|a_{i}\right| \\
\geq \gamma_{2} \sum_{i=(j-2) M}^{(j-1) M-1}\left|a_{i}\right|+\frac{\gamma_{2}}{2} \sum_{i=(j-1) M}^{(j+k) M-1}\left|a_{i}\right| \geq \frac{\gamma_{2}}{2} \sum_{i=(j-2) M}^{(j+k) M-1}\left|a_{i}\right| .
\end{array}
$$

If some blocks directly following $\mathcal{A}_{j}$ all have "u" labels, say $\mathcal{A}_{j+1}, \ldots, \mathcal{A}_{j+s}$ all have "u", then, first, Lemma 7 gives a lower estimate for each pair of consecutive blocks of them and since

$$
\begin{aligned}
& S_{2}\left(a_{(j-2) M}, \ldots, a_{(j+s) M-1}\right) \\
& =S_{2}\left(\operatorname{block} \mathcal{A}_{j}\right)+S_{2}\left(\operatorname{block} \mathcal{A}_{j+1}\right)+\ldots+S_{2}\left(\text { block } \mathcal{A}_{j+s}\right)+\text { more } \\
& \geq \frac{\varepsilon_{1}}{3} \sum_{i=(j-2) M}^{j M-1}\left|a_{i}\right|+\varepsilon_{1} \sum_{j M}^{(j+1) M-1}\left|a_{i}\right|+\ldots+\varepsilon_{1} \sum_{(j+s-1) M}^{(j+s) M-1}\left|a_{i}\right| \geq \gamma_{2} \sum_{i=(j-2) M}^{(j+s) M-1}\left|a_{i}\right|
\end{aligned}
$$

with $\gamma_{2}=\varepsilon_{1} / 3$, we have a lower estimate for $\left(S_{1}+S_{2}+S_{3}\right)\left(a_{(j-2) M}, \ldots\right.$ $\left.\ldots, a_{(j+s) M-1}\right)$.

Finally, suppose the $a_{i}$ 's go down in the first $j$ blocks $\mathcal{A}_{0}, \mathcal{A}_{1}, \ldots, \mathcal{A}_{j-1}$, and go up in $\mathcal{A}_{j}$. That is, we now consider all blocks before the first "u". Then, by Lemmas 6 and 7 there are $\gamma_{3}>0$ and $\gamma_{2}>0$ such that

$$
\begin{gathered}
\left(S_{1}+S_{2}+S_{3}\right)\left(a_{0}, \ldots, a_{(j-1) M-1}\right) \geq \gamma_{3} \sum_{i=0}^{(j-1) M-1}\left|a_{i}\right|, \\
\left(S_{1}+S_{2}+S_{3}\right)\left(a_{(j-2) M}, \ldots, a_{j M-1}\right) \geq \gamma_{2} \sum_{i=(j-2) M}^{j M-1}\left|a_{i}\right| .
\end{gathered}
$$

Then

$$
\begin{aligned}
& \left(S_{1}+S_{2}+S_{3}\right)\left(a_{0}, \ldots, a_{j M-1}\right) \\
& \quad=\left(S_{1}+S_{2}\right)\left(a_{0}, \ldots, a_{(j-1) M-1}\right)+S_{2}\left(a_{(j-2) M}, \ldots, a_{j M-1}\right)+\text { more } \\
& \quad \geq \gamma_{3} \sum_{i=0}^{(j-2) M-1}\left|a_{i}\right|+\gamma_{2} \sum_{i=(j-2) M}^{j M-1}\left|a_{i}\right| \geq \min \left\{\gamma_{2}, \gamma_{3}\right\} \sum_{i=0}^{j M-1}\left|a_{i}\right| .
\end{aligned}
$$

Subcases I.1 and I.2 prove the following lemma:

LEMMA 8. If the vector $w=\left(w_{1}, \ldots, w_{3 n-4}\right)$ gives $3 n-5$ decreasing geometric progressions $g^{1}, \ldots, g^{3 n-5}$, then there exists a $\gamma>0$, depending only on those progressions, such that

$$
\left(S_{1}+S_{2}+S_{3}\right)\left(a_{0}, \ldots, a_{m}\right) \geq \gamma \sum_{i=0}^{m}\left|a_{i}\right| .
$$


Thus, the equivalence from Theorem 2 is proved assuming that Case I occurs.

CASE II. All the previous lemmas work similarly in the case when $\left|y_{k}\right|>1$ for all $k=1,2, \ldots, 3 n-5$. The length $M$ of the blocks is determined by the following lemma, which we only state.

Lemma 9. There exists an $M>0$, depending only on the $3 n-5$ increasing geometric progressions $g^{1}, \ldots, g^{3 n-5}$, such that if $\left(\alpha_{i}\right) \in l^{1}$ can be written as $\left(\alpha_{i}\right)_{i \in \mathbb{N}}=\left(x_{1} g^{1}+\ldots+x_{3 n-5} g^{3 n-5}\right)$, where $g^{k}=\left(1, y_{k}, y_{k}^{2}, \ldots\right)$, $1 \leq k \leq 3 n-5$, with $\left|y_{k}\right|>1$ for all $k=1, \ldots, 3 n-5$, then

$$
\sum_{k=M}^{2 M-1}\left|\alpha_{k}\right| \geq 10 \sum_{k=0}^{M-1}\left|\alpha_{k}\right| .
$$

If $\left(\alpha_{k}\right) \in l^{1}$ can be written as a sum of $3 n-5$ increasing geometric progressions, then there exists an $m_{0} \in \mathbb{N}$ such that $\alpha_{m_{0}}=\alpha_{m_{0}+1}=\ldots=0$. Also, if $S_{2}\left(\alpha_{0}, \ldots, \alpha_{m}\right)=0$, the sum $\sum_{k=0}^{m}\left|\alpha_{k}\right|$ is determined by the last $M$-block of the $\alpha_{k}$ 's and the whole estimate of $S_{1}+S_{2}+S_{3}$ depends now on its third part $S_{3}\left(\alpha_{m-3 n+6}, \ldots, \alpha_{m}\right)$. The next lemma will state this result. The proof is similar to that of Lemma 6 .

Lemma 10. Fix $w=\left(w_{1}, \ldots, w_{3 n-4}\right)$ with $w_{1} \neq 0, w_{3 n-4} \neq 0$. For any sequence $\left(\alpha_{k}\right)$ of complex numbers with $0 \neq \sum\left|\alpha_{k}\right|<\infty$, let $A_{i}=$ $\left(\alpha_{i}, \alpha_{i+1}, \ldots, \alpha_{i+3 n-5}\right), i=0,1, \ldots$ If $\left\langle w, A_{i}\right\rangle=0$ for all $i=0,1, \ldots$, $m-3 n+6, m \in \mathbb{N}$, implies that $\left(\alpha_{0}, \alpha_{1}, \ldots, \alpha_{m}\right)$ can be written as a sum of $3 n-5$ progressions, then there is a $\delta_{2}>0$, depending only on those increasing geometric progressions, such that for any $m \geq 3 n-5$,

$$
\begin{aligned}
S_{3}\left(\alpha_{m-3 n+6}, \ldots, \alpha_{m}\right)= & \left|\alpha_{m-3 n+6} w_{1}+\alpha_{m-3 n+7} w_{2}+\ldots+\alpha_{m} w_{3 n-5}\right| \\
& +\left|\alpha_{m-3 n+7} w_{1}+\alpha_{m-3 n+8} w_{2}+\ldots+\alpha_{m} w_{3 n-6}\right| \\
& +\ldots+\left|\alpha_{m-1} w_{1}+a_{m} w_{2}\right|+\left|\alpha_{m} w_{1}\right| \geq \delta_{2} .
\end{aligned}
$$

CASE III. Next, suppose the geometric progressions $g^{i}=\left(1, y_{i}, y_{i}^{2}, \ldots\right)$, $i=1, \ldots, 3 n-5$, that we get by Proposition 3 satisfy $\left|y_{i}\right|<1$ for $1 \leq i \leq l$ and $\left|y_{i}\right|>1$ for $l+1 \leq i \leq 3 n-5$. So, if $S_{2}\left(a_{0}, \ldots, a_{m}\right)=0$, then by Proposition $3,\left(a_{0}, \ldots, a_{m}\right)$ is an exact linear combination of the geometric progressions $g^{1}, \ldots, g^{3 n-5}$, that is,

$$
\left(a_{0}, \ldots, a_{m}\right)=\left(x_{1} g^{1}+\ldots+x_{l} g^{l}\right)+\left(x_{l+1} g^{l+1}+\ldots+x_{3 n-5} g^{3 n-5}\right) .
$$

Consider first the decreasing progressions $g^{1}, \ldots, g^{l}$ only. For these progressions Lemma 4 determines a block length $M$ so that 


$$
\sum_{k=M}^{2 M-1}\left|a_{k}\right| \leq \frac{1}{10} \sum_{k=0}^{M-1}\left|a_{k}\right|
$$

for all sequences $\left(a_{k}\right) \in l^{1}$ that can be written as $\left(a_{k}\right)=x_{1} g^{1}+\ldots+x_{l} g^{l}$ for some complex numbers $x_{1}, \ldots, x_{l}$.

Using this $M$, divide any $\left(a_{0}, \ldots, a_{m}\right)$ into blocks of length $M$, and in every block redetermine the coefficients $x_{1}, \ldots, x_{3 n-5}$ in its representation by $3 n-5$ geometric progressions. For all those blocks that are exact linear combinations of the first $l$ decreasing geometric progressions $g^{1}, \ldots, g^{l}$, we have a $\gamma>0$, depending only on $g^{1}, \ldots, g^{l}$, and thus a lower estimate by Lemma 8 of Case I.

We now fix a projection from the space of $M$-tuples $\left(a_{0}, \ldots, a_{M-1}\right) \in \mathbb{C}^{M}$ onto $\overline{\operatorname{span}}\left\{g^{1}, \ldots, g^{3 n-5}\right\}$. Hence, in each $M$-block we can project $\left(a_{j M}, \ldots\right.$ $\left.\ldots, a_{(j+1) M-1}\right)$ onto that span, that is, we have $\left(a_{j M}, \ldots, a_{(j+1) M-1}\right)=$ $x_{1} g^{1}+\ldots+x_{3 n-5} g^{3 n-5}+\eta$, where $\eta$ has the smallest possible norm. We also consider the projections $P_{\text {first }}$ and $P_{\text {last }}$ that project $\left(a_{j M}, \ldots, a_{(j+1) M-1}\right)$ onto $\overline{\operatorname{span}}\left\{g^{1}, \ldots, g^{l}\right\}$ and $\overline{\operatorname{span}}\left\{g^{l+1}, \ldots, g^{3 n-5}\right\}$ respectively.

In Lemmas 11 through 16 we consider the sequences $\left(a_{k}\right)$ that are exact linear combinations of geometric progressions $g^{1}, \ldots, g^{3 n-5}$ in each block. That is, in every block we have $\eta=0$. The general case will be considered in Lemma 17.

LEMMA 11. Given $a_{0}, \ldots, a_{m}$ and a block length $M$, if

$$
\sum_{j=1}^{[|(m+1) / M|]}\left\|P_{\text {last }}\left(a_{(j-1) M}, \ldots, a_{j M-1}\right)\right\| \leq \frac{\gamma}{2} \sum_{k=0}^{m}\left|a_{k}\right|,
$$

then there is a $\gamma_{4}>0$, depending only on $g^{1}, \ldots, g^{3 n-5}$, such that

$$
S_{1}+S_{2}+S_{3} \geq \gamma_{4} \sum_{k=0}^{m}\left|a_{k}\right| \text {. }
$$

Proof. Since

$$
\begin{aligned}
S_{1}\left(a_{0}, \ldots, a_{3 n-6}\right)+ & S_{2}\left(a_{0}, \ldots, a_{m}\right)+S_{3}\left(a_{m-3 n+6}, \ldots, a_{m}\right) \\
\geq & S_{1}\left(P_{\text {first }}\left(a_{0}, \ldots, a_{3 n-6}\right)\right)+S_{2}\left(P_{\text {first }}\left(a_{0}, \ldots, a_{m}\right)\right) \\
& \left.+S_{3}\left(P_{\text {first }}\left(a_{m-3 n+6}, \ldots, a_{m}\right)\right)\right] \\
& -\left[S_{1}\left(P_{\text {last }}\left(a_{0}, \ldots, a_{3 n-6}\right)\right)+S_{2}\left(P_{\text {last }}\left(a_{0}, \ldots, a_{m}\right)\right)\right. \\
& \left.+S_{3}\left(P_{\text {last }}\left(a_{m-3 n+6}, \ldots, a_{m}\right)\right)\right] \\
\geq \gamma & \sum_{k=0}^{m}\left|P_{\text {first }}\left(a_{k}\right)\right|-\sum_{k=0}^{m}\left|P_{\text {last }}\left(a_{k}\right)\right|
\end{aligned}
$$




$$
\begin{aligned}
& \geq \gamma \sum_{k=0}^{m}\left|a_{k}\right|-\gamma \sum_{k=0}^{m}\left|P_{\text {last }}\left(a_{k}\right)\right|-\sum_{k=0}^{m}\left|P_{\text {last }}\left(a_{k}\right)\right| \\
& \geq\left(\gamma-\frac{\gamma^{2}}{2}-\frac{\gamma}{2}\right) \sum_{k=0}^{m}\left|a_{k}\right|=\gamma_{4} \sum_{k=0}^{m}\left|a_{k}\right|
\end{aligned}
$$

the lemma is proved.

LEMMA 12. If

$$
\sum_{j=1}^{[|(m+1) / M|]}\left\|P_{\text {last }}\left(a_{(j-1) M}, \ldots, a_{j M-1}\right)\right\|>\frac{\gamma}{2} \sum_{k=0}^{m}\left|a_{k}\right|,
$$

then there is at least one block $\mathcal{A}_{j_{0}}$ of the $a_{k}$ 's such that

$$
\left\|P_{\text {last }}\left(a_{\left(j_{0}-1\right) M}, \ldots, a_{j_{0} M-1}\right)\right\| \geq \frac{\gamma}{10} \sum_{k=\left(j_{0}-1\right) M}^{j_{0} M-1}\left|a_{k}\right| .
$$

Proof. If not, that is, when in all blocks we have

$$
\left\|P_{\text {last }}\left(a_{(j-1) M}, \ldots, a_{j M-1}\right)\right\|<\frac{\gamma}{10} \sum_{k=(j-1) M}^{j M-1}\left|a_{k}\right|,
$$

$j=1, \ldots, m-M$, then

$$
\sum\left\|P_{\text {last }}\left(a_{(j-1) M}, \ldots, a_{j M-1}\right)\right\|<\frac{\gamma}{10} \sum_{k=0}^{m}\left|a_{k}\right|,
$$

contradicting the hypothesis.

LEMMA 13. The weight of the blocks with

$$
\left\|P_{\text {last }}\left(a_{(j-1) M}, \ldots, a_{j M-1}\right)\right\| \geq \frac{\gamma}{10} \sum_{k=(j-1) M}^{j M-1}\left|a_{k}\right|
$$

is at least

$$
\frac{\gamma}{10} \sum_{k=0}^{m}\left|a_{k}\right|
$$

The next lemma determines a new block length:

Lemma 14. There exist $n_{1} \in \mathbb{N}$ and $\gamma_{5}>0$, depending only on $g^{1}, \ldots$ $\ldots, g^{3 n-5}$, such that if

$$
\sum_{k=1}^{n_{1}}\left\|P_{\text {last }}\left(a_{(j+k-1) M}, \ldots, a_{(j+k) M-1}\right)\right\| \geq \frac{\gamma}{10} \sum_{i=j M}^{\left(j+n_{1}\right) M-1}\left|a_{i}\right|,
$$


then either

$$
\sum_{k=1}^{n_{1}}\left\|P_{\text {last }}\left(a_{\left(\left(j+n_{1}\right)+k-1\right) M}, \ldots, a_{\left(\left(j+n_{1}\right)+k\right) M-1}\right)\right\| \geq \frac{\gamma}{10} \sum_{i=\left(j+n_{1}\right) M}^{\left(j+2 n_{1}\right) M-1}\left|a_{i}\right|
$$

and

$$
\sum_{i=\left(j+n_{1}\right) M}^{\left(j+2 n_{1}\right) M-1}\left|a_{i}\right| \geq 10 \sum_{i=j M}^{\left(j+n_{1}\right) M-1}\left|a_{i}\right|
$$

or

$$
\left(S_{1}+S_{2}+S_{3}\right)\left(a_{j M}, \ldots, a_{\left(j+2 n_{1}\right) M-1}\right) \geq \gamma_{5} \sum_{i=j M}^{\left(j+2 n_{1}\right) M-1}\left|a_{i}\right| .
$$

Proof. If the first assertion does not hold, then the $a_{k}$ 's cannot be written as exact linear combinations of $3 n-5$ geometric progressions. So, by a compactness argument as in Lemma 6 , there is a $\gamma_{5}>0$ such that

$$
\left(S_{1}+S_{2}+S_{3}\right)\left(a_{0}, \ldots, a_{2 n_{1} M-1}\right) \geq \gamma_{5} \sum_{k=0}^{2 n_{1} M-1}\left|a_{k}\right| .
$$

Note that when $\left(a_{k}\right)$ is close to $\overline{\operatorname{span}}\left\{g^{1}, \ldots, g^{3 n-5}\right\}$, the first assertion holds.

Call these new blocks $\mathcal{B}_{1}, \mathcal{B}_{2}, \mathcal{B}_{3}, \ldots$ Each of them has now the block length $n_{1} M$.

DEFINITION 15. Let

$$
\left\|P_{\text {last }}\left(\mathcal{B}_{j}\right)\right\| \geq \frac{\gamma}{10} \sum_{a_{k} \in \mathcal{B}_{j}}\left|a_{k}\right| .
$$

If

$$
\left\|P_{\text {last }}\left(\mathcal{B}_{j+1}\right)\right\| \geq \frac{\gamma}{10} \sum_{a_{k} \in \mathcal{B}_{j+1}}\left|a_{k}\right|
$$

and $\left\|\mathcal{B}_{j+1}\right\| \geq 2\left\|\mathcal{B}_{j}\right\|$, then we say that they go up from the block $\mathcal{B}_{j}$. Otherwise they go down from $\mathcal{B}_{j}$.

We now consider blocks with the $P_{\text {last }}$ projections onto the subspace $\overline{\operatorname{span}}\left\{g^{l+1}, \ldots, g^{3 n-5}\right\}$ having norm greater than $\gamma / 10$ of the sum of the $a_{k}$ 's in that block. It is enough to have a lower estimate of $\sum_{k=0}^{m}\left|a_{k}\right|$ in terms of the $a_{k}$ 's from the blocks with $\left\|P_{\text {last }}\right\|$ large, since, by Lemma 13 , these blocks have big weight. Under each of these blocks we write "u" if the $a_{k}$ 's go up from that block, and "d" otherwise. Labeling only the blocks with $\left\|P_{\text {last }}\left(\mathcal{B}_{j}\right)\right\| \geq \frac{\gamma}{10} \sum_{a_{k} \in \mathcal{B}_{j}}\left|a_{k}\right|$, we also have, in general, blocks without a label, namely the ones that have $\left\|P_{\text {last }}\left(\mathcal{B}_{j}\right)\right\|<\frac{\gamma}{10} \sum_{a_{k} \in \mathcal{B}_{j}}\left|a_{k}\right|$. In this way the whole sequence $\left(a_{k}\right)$ is divided into blocks where blocks having a label 
are separated by the blocks without a label. It is enough to have a lower estimate of $S_{1}+S_{2}+S_{3}$ for each sequence of consecutive labeled blocks.

So, consider a sequence of blocks $\mathcal{B}_{j}, \mathcal{B}_{j+1}, \ldots, \mathcal{B}_{j+l}$, in which every block has a label but $\mathcal{B}_{j-1}$ and $\mathcal{B}_{j+l+1}$ are without a label. Observe that each such sequence of blocks $\mathcal{B}_{j}, \ldots, \mathcal{B}_{j+l}$ ends with a "d" label.

We have the following estimates depending on the order of labels: By Lemma 14, we always have a lower estimate for blocks $\mathcal{B}_{i}$ and $\mathcal{B}_{i+1}$ if $\mathcal{B}_{i}$ has label "d". We also have an estimate for all previous blocks with " $\mathrm{u}$ ", that is, if $\mathcal{B}_{i-s}, \ldots, \mathcal{B}_{i-1}$ have "u" and $\mathcal{B}_{i}$ has "d", then since the $a_{k}$ 's in the block $\mathcal{B}_{i}$ dominate, that is,

$$
\sum_{k=(i-s-1) n_{1} M}^{i n_{1} M-1}\left|a_{k}\right| \leq 2 \sum_{k=(i-1) n_{1} M}^{i n_{1} M-1}\left|a_{k}\right|,
$$

we have

$$
\begin{aligned}
& \left(S_{1}+S_{2}+S_{3}\right)\left(a_{(i-s-1) n_{1} M}, \ldots, a_{(i+1) n_{1} M-1}\right) \\
& \geq \gamma_{5} \sum_{k=(i-1) n_{1} M}^{i n_{1} M-1}\left|a_{k}\right|+\gamma_{5} \sum_{k=i n_{1} M}^{(i+1) n_{1} M-1}\left|a_{k}\right| \\
& \geq \frac{\gamma_{5}}{2} \sum_{k=(i-s-1) n_{1} M}^{i n_{1} M-1}\left|a_{k}\right|+\gamma_{5} \sum_{k=i n_{1} M}^{(i+1) n_{1} M-1}\left|a_{k}\right| \geq \frac{\gamma_{5}}{2} \sum_{k=(i-s-1) n_{1} M}^{(i+1) n_{1} M-1}\left|a_{k}\right| .
\end{aligned}
$$

When the $a_{i}$ 's have label "d" in two or more consecutive blocks, say in $\mathcal{B}_{i}, \ldots, \mathcal{B}_{i+s}$, then, by a compactness argument, in each pair of consecutive blocks and by the form of the sum $S_{2}\left(a_{(i-1) n_{1} M}, \ldots, a_{(i+s) n_{1} M-1}\right)$ in terms of the sums $S_{2}$ (block $\left.\mathcal{B}_{i}\right), S_{2}$ (block $\left.\mathcal{B}_{i+1}\right), \ldots, S_{2}$ (block $\left.\mathcal{B}_{i+s}\right)$, we have also a lower estimate (compare with the proof of the second half of Subcase I.2 of Case I).

So, if a vector $w=\left(w_{1}, \ldots, w_{3 n-4}\right)$ gives us, by Proposition $3,3 n-5$ geometric progressions $g^{1}, \ldots, g^{3 n-5}$, where $g^{1}, \ldots, g^{l}$ are decreasing and $g^{l+1}, \ldots, g^{3 n-5}$ are increasing, then the previous discussion proves the following:

Lemma 16. There exists a $\gamma^{\prime}>0$, depending only on the geometric progressions $g^{1}, \ldots, g^{3 n-5}$, such that for any sequence $\left(a_{k}\right) \in l^{1}$ and for any $m \in \mathbb{N}$ we have

$$
\left(S_{1}+S_{2}+S_{3}\right)\left(a_{0}, \ldots, a_{m}\right) \geq \gamma^{\prime} \sum_{k=0}^{m}\left|a_{k}\right| .
$$

Finally, for a general sequence $\left(a_{k}\right)$, in each block write it as $\left(a_{k}\right)=$ $x_{1} g^{1}+\ldots+x_{l} g^{l}+x_{l+1} g^{l+1}+\ldots+x_{3 n-5} g^{3 n-5}+\eta$ with $\left(\eta^{i}\right)$ having a minimal 
$l^{1}$-norm. Then the following lemma finishes the case of mixed geometric progressions:

Lemma 17. There exists a $\gamma_{6}>0$, depending only on $g^{1}, \ldots, g^{3 n-5}$, such that if in every block $\mathcal{B}_{i}, i=1,2, \ldots$, a sequence $\left(a_{k}\right)$ can be written as $x_{1}^{i} g_{1}+\ldots+x_{l}^{i} g_{l}+x_{l+1}^{i} g_{l+1}+\ldots+x_{3 n-5}^{i} g_{3 n-5}+\eta^{i}$ then for any $m \in \mathbb{N}$,

$$
S_{1}+S_{2}+S_{3} \geq \gamma_{6} \sum_{k=0}^{m}\left|a_{k}\right| \text {. }
$$

Proof. The case $\eta=0$ is covered by previous lemmas. Let $S_{1}^{\prime}, S_{2}^{\prime}$, and $S_{3}^{\prime}$ denote the sums in the case of $\eta=0$, and let $\gamma^{*}>0$ be such that $S_{1}^{\prime}+S_{2}^{\prime}+S_{3}^{\prime} \geq \gamma^{*} \sum_{k=0}^{m}\left|a_{k}\right|$. If $\|\eta\|=\sum\left\|\eta^{i}\right\| \leq\left(\gamma^{*} / 2\right) \sum\left|a_{k}\right|$, then $S_{1}+S_{2}+S_{3} \geq\left(S_{1}^{\prime}+S_{2}^{\prime}+S_{3}^{\prime}\right)-\|\eta\| \geq \gamma^{*} \sum_{k=0}^{m}\left|a_{k}\right|-\frac{\gamma^{*}}{2} \sum_{k=0}^{m}\left|a_{k}\right|=\frac{\gamma^{*}}{2} \sum_{k=0}^{m}\left|a_{k}\right|$, that is, $\gamma_{6}=\gamma^{*} / 2$. If $\|\eta\|>\left(\gamma^{*} / 2\right) \sum\left|a_{k}\right|$, then $\left(a_{k}\right)$ is far from being a linear combination of $3 n-5$ geometric progressions and the estimate follows using a compactness argument.

Thus, the stated equivalence is also proved assuming Case III occurs.

Remark 1. Suppose $\left|y_{i}\right|=1,1 \leq i \leq 3 n-5$. Then there always exists a simple function whose orbit under the isometry $T$ does not produce a sequence equivalent to the $l^{1}$-canonical basis. For example, let $\lambda=1 / 2$, and let

$$
s(t)= \begin{cases}a & \text { on }[0,1 / 2), \\ b & \text { on }[1 / 2,1] .\end{cases}
$$

Then for $a=-b$ or $a=3 b$ the orbit of $s$ under $T$ with $\lambda=1 / 2$ is not equivalent to the usual basis of $l^{1}$.

More generally, if $s$ can be written as

$$
s=g-\frac{1}{k}\left(T^{m_{1}} g-T^{m_{2}} g-\ldots-T^{m_{k}} g\right)
$$

for some $g \in L^{1}$ and $k \in \mathbb{N}$, then $\left\|\sum_{i=0}^{m} a_{i} T^{i} s\right\|$ is bounded for any $m$, but $\sum_{i=0}^{m}\left|a_{i}\right|=\sum_{i=0}^{m}$ const $=(m+1)$ const $\rightarrow \infty$ as $m \rightarrow \infty$. Hence no lower estimate exists. But we can always change those "bad" simple functions by some $\widehat{\varepsilon}>0$ and so the result still holds for a dense set of functions in $L^{1}$.

REMARK 2 (Shift operator on $l^{1}$ ). We define the shift operator on $l^{1}$ by $T\left(\left(x_{1}, x_{2}, x_{3}, \ldots\right)\right)=\left(0, x_{1}, x_{2}, x_{3}, \ldots\right)$. Then we also have the following result:

THEOREM 18. The set of sequences in $l^{1}$ whose orbit under the shift operator $T$ is equivalent to the usual basis of $l^{1}$ is an open dense set. 
Proof. Since the sequences with finitely many nonzero coordinates are dense in $l^{1}$, for any fixed $n \in \mathbb{N}$ and $x=\left(x_{1}, \ldots, x_{n}, 0,0, \ldots\right) \in l^{1}$ we have

$$
\begin{aligned}
&\left\|\sum_{k=0}^{m} a_{k} T^{k} x\right\| \\
&=\mid\left|a_{0} x_{1}\right|+\left|a_{0} x_{2}+a_{1} x_{1}\right|+\left|a_{0} x_{3}+a_{1} x_{2}+a_{2} x_{1}\right| \\
&+\ldots+\left|a_{0} x_{n-1}+a_{1} x_{n-2}+\ldots+a_{n-2} x_{1}\right| \\
&+\left|a_{0} x_{n}+a_{1} x_{n-1}+\ldots+a_{n-1} x_{1}\right|+\left|a_{1} x_{n}+a_{2} x_{n-1}+\ldots+a_{n} x_{1}\right| \\
&+\ldots+\left|a_{m-n+1} x_{n}+a_{m-n+2} x_{n-1}+\ldots+a_{m} x_{1}\right| \\
&+\left|a_{m-n+2} x_{n}+\ldots+a_{m} x_{2}\right|+\ldots+\left|a_{m-1} x_{n}+a_{m} x_{n-1}\right|+\left|a_{m} x_{n}\right| \\
&= S_{1}\left(a_{0}, \ldots, a_{n-2}\right)+S_{2}\left(a_{0}, \ldots, a_{m}\right)+S_{3}\left(a_{m-n+2}, \ldots, a_{m}\right),
\end{aligned}
$$

for any $m \in \mathbb{N}$ and $\left(a_{k}\right) \in l^{1}$. Thus, we have to estimate the sum $S_{1}+S_{2}+S_{3}$. The rest of the proof is similar to that of Theorem 2 .

Note again that if $\left|y_{i}\right|=1$ for some $i$, then taking $a_{0}=\ldots=a_{m}=1$ we have $\left\|\sum T^{k} x\right\|=$ finite, but $\sum a_{k}=m+1 \rightarrow \infty$, i.e. the orbit of such an $x$ is not equivalent to the usual basis of $l^{1}$.

REMARK 3. Theorem 18 is also partially true in any other $l^{p}, 1<p<\infty$. That is, for any $n \in \mathbb{N}$ and $x=\left(x_{1}, \ldots, x_{n}, 0, \ldots\right) \in l^{p}$ with $\|x\|_{p}=1$, we get the estimates

$$
\gamma\left(\sum_{k=0}^{m}\left|a_{k}\right|^{p}\right)^{1 / p} \leq\left\|\sum_{k=0}^{m} a_{k} T^{k} x\right\|_{p} \leq n\left(\sum_{k=0}^{m}\left|a_{k}\right|^{p}\right)^{1 / p} .
$$

The upper estimate is clear; the lower estimate follows from compactness arguments as in Theorems 2 and 18. This yields

TheOREM 19. Every $l^{p}, 1<p<\infty$, contains a dense set of sequences whose orbit under the shift operator $T$ is equivalent to the usual basis of $l^{p}$.

The only difference with Theorem 18 is that we do not know whether this dense set is open or not when $1<p<\infty$. Recall that it was open in $l^{1}$.

Remark 4. Define

$$
\begin{aligned}
& \underset{\theta}{\operatorname{essinf}}\left|f\left(e^{i \theta}\right)\right|=\inf \{A: m\{z:|f(z)|<A\}>0\}, \\
& \underset{\theta}{\operatorname{ess} \sup }\left|f\left(e^{i \theta}\right)\right|=\sup \{B: m\{z:|f(z)|>B\}>0\} .
\end{aligned}
$$

Then we have the following result in $H^{2}$ :

THEOREM 20. Let the operator $T$ be multiplication by $z$ on $H^{2}$. Then the orbit of $f \in H^{2}$ under $T$, that is, the set $\left\{f, z f, z^{2} f, \ldots\right\}$, is equivalent to the usual basis of $l^{2}$ if and only if $\operatorname{ess} \inf _{\theta}\left|f\left(e^{i \theta}\right)\right|>0$ and $\operatorname{ess}_{\sup }\left|f\left(e^{i \theta}\right)\right|<\infty$. 
Proof. Clearly, we have

$$
\begin{aligned}
\underset{\theta}{\operatorname{essinf}}\left|f\left(e^{i \theta}\right)\right|\left(\sum_{k=0}^{m}\left|a_{k}\right|^{2}\right)^{1 / 2} & \leq\left\|\sum_{k=0}^{m} a_{k} z^{k} f\right\|_{2} \\
& \leq \underset{\theta}{\operatorname{ess} \sup }\left|f\left(e^{i \theta}\right)\right|\left(\sum_{k=0}^{m}\left|a_{k}\right|^{2}\right)^{1 / 2},
\end{aligned}
$$

and if $\operatorname{ess}_{\inf }\left|f\left(e^{i \theta}\right)\right|>0$ and $\operatorname{ess}_{\sup }\left|f\left(e^{i \theta}\right)\right|<\infty$, the estimate follows.

For the sufficiency, suppose instead that $\operatorname{essinf}_{\theta}\left|f\left(e^{i \theta}\right)\right|=0$. By the definition, this means that for any $\varepsilon>0$ there is an interval $(\alpha, \beta) \subseteq$ $\left\{\theta:\left|f\left(e^{i \theta}\right)\right| \leq \varepsilon\right\}$. We can suppose $0 \in(\alpha, \beta)$ and define a peak function $\varphi\left(e^{i \theta}\right)$ by $\varphi\left(e^{i \theta}\right)=\sum_{k=1}^{\infty} a_{k} e^{i k \theta}$ with $\operatorname{supp} \varphi \subseteq(\alpha, \beta)$ and $\|\varphi\|_{2}=1$. Then

$$
\begin{aligned}
\left\|\sum_{k=1}^{m} a_{k} z^{k} f\right\|_{2}^{2} & =\frac{1}{2 \pi} \int_{0}^{2 \pi}\left|\sum_{k=1}^{m} a_{k} e^{i k \theta} f\left(e^{i \theta}\right)\right|^{2} d \theta \\
& \leq \frac{1}{2 \pi} \int_{\alpha}^{\beta} \varepsilon^{2}\left|\varphi\left(e^{i \theta}\right)\right|^{2} d \theta=\frac{\varepsilon^{2}}{2 \pi} .
\end{aligned}
$$

Letting $\varepsilon \rightarrow 0$, we see that no constant $c_{1}>0$ exists that satisfies

$$
c_{1}\left(\sum_{k=1}^{m}\left|a_{k}\right|^{2}\right)^{1 / 2} \leq\left\|\sum_{k=1}^{m} a_{k} z^{k} f\right\|_{2} .
$$

The proof for an upper constant $c_{2}$ is similar.

REMARK 5 (A characterization of functions in $A(\mathbb{T})$ ). We denote by $A(\mathbb{T})$ the set of all continuous complex-valued functions $f$ on $\mathbb{T}$ which have absolutely convergent Fourier series. If $f(z)=\sum_{k=-\infty}^{\infty} c_{k} z^{k}$, then its norm in $A(\mathbb{T})$ is defined by $\|f\|_{A}=\sum_{k=-\infty}^{\infty}\left|c_{k}\right|$. We have the following characterization:

THEOREM 21. Let the operator $M$ be multiplication by $z$. Then the orbit of $f \in A(\mathbb{T})$ under $M$, that is, the set $\left\{f, z f, z^{2} f, \ldots\right\}$, is equivalent to the usual basis of $l^{1}$ if and only if $f$ has no zeros on the unit circle.

Proof. Wiener's Theorem says that if $f$ has absolutely convergent Fourier series and has no zeros on the boundary, then $1 / f$ has absolutely convergent Fourier series. Using this theorem we can write

$$
\left\|\sum_{k=0}^{m} a_{k} z^{k}\right\|=\left\|\frac{1}{f} \cdot f \sum_{k=0}^{m} a_{k} z^{k}\right\| \leq\left\|\frac{1}{f}\right\|\left\|\sum_{k=0}^{m} a_{k} z^{k} f\right\|
$$

and thus

$$
\frac{1}{\|1 / f\|}\left\|\sum_{k=0}^{m} a_{k} z^{k}\right\| \leq\left\|\sum_{k=0}^{m} a_{k} z^{k} f\right\|
$$


that is,

$$
\frac{1}{\|1 / f\|} \sum_{k=0}^{m}\left|a_{k}\right| \leq\left\|\sum_{k=0}^{m} a_{k} z^{k} f\right\| .
$$

The last inequality with $\gamma=1 /\|1 / f\|$ proves the sufficiency part of the theorem.

Suppose now $f(z)=\sum_{k=-\infty}^{\infty} c_{k} z^{k}$ in $A(\mathbb{T})$ has a zero on the unit circle. We may suppose the zero is at $z=1$. Then $\sum_{k=-\infty}^{\infty} c_{k}=0$. Fix $\varepsilon>0$ and find $n \in \mathbb{N}$ such that $\sum_{k=-\infty}^{-(n+1)}\left|c_{k}\right|+\sum_{k=n+1}^{\infty}\left|c_{k}\right|<\varepsilon$. Let $p(z)=$ $\sum_{k=-n}^{n} c_{k} z^{k}+b_{n+1} z^{n+1}$ with $b_{n}=\sum_{k=-\infty}^{-(n+1)} c_{k}+\sum_{k=n+1}^{\infty} c_{k}$ be a polynomial with a zero at $z=1$. Then, if $a_{0}=a_{1}=\ldots=a_{m}=1$, we have

$$
\left\|\sum_{k=-m}^{m} z^{k} f\right\| \leq\left\|\sum_{k=-m}^{m} z^{k} p\right\|+\left\|\sum_{k=-m}^{m} z^{k}(f-p)\right\| .
$$

Since, by Theorem 18, $\left\|\sum_{k=-m}^{m} z^{k} p\right\|$ is finite and independent of $m$, and since $\|f-p\|<3 \varepsilon$, we get

$$
\left\|\sum_{k=-m}^{m} z^{k} f\right\| \leq \text { const }+(2 m+1) \cdot 3 \varepsilon .
$$

Since the last inequality is true for every $\varepsilon$, we see that there is no $\gamma>0$ such that

$$
\gamma \sum_{k=-m}^{m}\left|a_{k}\right| \leq\left\|\sum_{k=-m}^{m} a_{k} z^{k} f\right\|
$$

for all $a_{0}, \ldots, a_{m}$ and all $m \in \mathbb{N}$.

Open Questions. 1. When is the orbit of a cyclic vector $x$ in $l^{1}$ a basis for $l^{1}$ ?

By Theorem 18, there is a dense set of cyclic vectors in $l^{1}$ whose orbit is a basic sequence (that is, a basis in the closure of its linear span). Our conjecture is that for $\|x\|=1$, the sequence $\left\{x, T x, T^{2} x, \ldots\right\}$, where $T$ is the shift operator, is a basis for $l^{1}$ if either $x=e_{1}$ or $x=\left(x_{1}, \ldots, x_{n}, 0,0, \ldots\right)$ is a linear combination of decreasing geometric progressions.

2. How large is the class of isometries of $L^{1}[0,1]$ to which Theorem 2 applies? It seems that the theorem is true for a more general class of isometries that "squeeze" and "stretch" measurable sets.

3 . Is the set of cyclic vectors in $L^{1}[0,1]$ or $l^{1}$ first category?

4. "How much" can one remove from these orbits and still have a spanning set? A similar question: When is the orbit of a cyclic vector a minimal spanning set?

5. A more general question about $L^{1}[0,1]$ : Can we find an operator and a cyclic vector whose orbit is a (normalized) Schauder basis? 
T. Hõim

\section{References}

[1] B. Beauzamy, Introduction to Operator Theory and Invariant Subspaces, NorthHolland, 1988.

[2] M. I. Kadec and A. Pełczyński, Bases, lacunary sequences and complemented subspaces in the spaces $L_{p}$, Studia Math. 21 (1962), 161-176.

[3] J. Lamperti, On the isometries of certain function-spaces, Pacific J. Math. 8 (1958), 459-466.

Department of Mathematics

Kent State University

Kent, OH 44240, U.S.A.

E-mail: terje.hoim@trincoll.edu

Received August 1, 2000

Revised version December 19, 2001 